\title{
Fully Automatic Liver Volumetry Using 3D Level Set Segmentation for Differentiated Liver Tissue Types in Multiple Contrast MR Datasets
}

\author{
Oliver Gloger ${ }^{1}$, Klaus Toennies ${ }^{2}$, and Jens-Peter Kuehn ${ }^{3}$ \\ 1 Ernst Moritz Arndt University of Greifswald, Institute for Community Medicine, \\ Walther-Rathenau-Str.48. 17475 Greifswald, Germany \\ gloger@uni-greifswald.de \\ 2 Otto-von-Guericke University of Magdeburg, Institute for Simulation and Graphics, \\ Universitaetsplatz 2, 39106 Magdeburg, Germany \\ klaus@isg. uni-magdeburg. de \\ 3 Ernst Moritz Arndt University of Greifswald, Institute for Diagnostic Radiology \\ and Neuroradiology, Ferdinand-Sauerbruch-Strasse, 17475 Greifswald, Germany \\ kuehn@uni-greifswald.de
}

\begin{abstract}
Modern epidemiological studies analyze a high amount of magnetic resonance imaging (MRI) data, which requires fully automatic segmentation methods to assist in organ volumetry. We propose a fully automatic two-step 3D level set algorithm for liver segmentation in MRI data that delineates liver tissue on liver probability maps and uses a distance transform based segmentation refinement method to improve segmentation results. MR intensity distributions in test subjects are extracted in a training phase to obtain prior information on liver, kidney and background tissue types. Probability maps are generated by using linear discriminant analysis and Bayesian methods. The algorithm is able to differentiate between normal liver tissue and fatty liver tissue and generates probability maps for both tissues to improve the segmentation results. The algorithm is embedded in a volumetry framework and yields sufficiently good results for use in epidemiological studies.
\end{abstract}

Keywords: Level Set Segmentation, Distance Transformation, Linear Discriminant Analysis, Bayes' Theorem.

\section{Introduction}

Advances in medical imaging techniques have substantially increased the demand on medical image segmentation. Besides being used as a tool in routine clinical practise, medical image segmentation can be very helpful for analyzing certain health-related properties in populations in modern epidemiological science. Organ volumetry is an important part of this. Epidemiological studies often analyze enormous amounts of data from participants, which would make manual segmentation very time-consuming and exhaustive and, consequently, prone to intra- and interreader bias.

A. Heyden and F. Kahl (Eds.): SCIA 2011, LNCS 6688, pp. 512 523, 2011.

(C) Springer-Verlag Berlin Heidelberg 2011 
Automatic image segmentation is preferred but is difficult because understanding the complexity of medical images requires radiologists with special training and skills. Automatic liver segmentation is particularly challenging since the properties of hepatic tissue are similar to those of adjacent organs and tissues. Consequently, state-of-the-art segmentation techniques can lead to oversegmentations with liver segments including adjacent organs such as the kidney, pancreas, spleen, and stomach. Conversely, some diseases such as cirrhosis, fatty liver, or tumors can show heterogeneous tissue properties inside the liver, which can lead to undersegmentation.

This work sets out to assist medical experts in analyzing MR datasets in the setting of epidemiological studies investigating large populations of different subjects. MR datasets typically show lower contrast and smaller edge magnitudes than CT datasets. Further challenges of tissue delineation in MR datasets are motion and pulsation artifacts as well as partial volume effects. All of these drawbacks require robust segmentation techniques especially designed for MR datasets and incorporating flexibilities for identifying oversegmentation in the setting of automatic volumetry. The approach presented here uses flexible level set segmentation including the ability of recognizing and reducing oversegmentation. Liver MR signal intensity patterns of multiple contrast datasets differ strongly between normal and fatty livers. The method proposed here differs from existing approaches for MR image segmentation in that it differentiates between fat liver and normal liver, enabling successful segmentation for both kinds of liver tissues.

\section{Related Work}

Literature research shows that numerous approaches for liver segmentation in CT datasets exist, while only a few methods have been proposed for MR datasets. CT segmentation approaches cover a wider spectrum of different methods ranging from fundamental concepts (like histogram analysis, morphological operations, threshold techniques, and region growing) [1, 2] to deformable models [3, 4, 5], atlas-based concepts [6, 7, 8, 9, classification methods [10, 11, 12, and graph-cut techniques [13, 14. There exist a fast liver segmentation method [15] especially for contrast enhanced MR images using a partitioned probabilistic model. However, only few approaches are available for native MR datasets using level set segmentation techniques [16, 17, 18] or fast marching and improved fuzzy cluster methods 19.

Although MR acquisition usually produces multiple contrast datasets, existing analysis approaches to the segmentation of MR datasets do not apply image information from all weighting available. Variations in appearance resulting from liver disease or the presence of different types of liver tissue are not accounted for. Twenty percent of the subjects in our study have fatty livers [20. Fatty livers have different tissue properties than nonfatty livers and therefore have different MR signal intensities. Automatic methods should recognize fatty livers and segment them as well as nonfatty livers. Hence, we developed a fully automatic liver 
segmentation framework that can differentiate between fatty livers and nonfatty livers and segments both types of tissue by using class-specific prior knowledge about MR signal intensities. We use 3D geodesic active contours and extend and accelerate the work of [21] for segmentation of liver-specific probability maps by calculating improved stopping terms in a two-step segmentation approach. The drawbacks of existing approaches discussed above are overcome by the approach we are presenting here.

\section{Data Acquisition}

The study is based on images taken from participants of the population-based Study of Health in Pomerania (SHIP). All abdominal MR datasets were acquired on a 1.5 Tesla MR scanner (Magnetom Avanto; Siemens Medical Systems, Erlangen, Germany). Subjects were placed in the supine position, and two phasedarray surface coils were placed over the abdomen and pelvis. The spinal coil was embedded in the scanner table. Two trained technicians performed all examinations in a standardized way. Four different datasets were created using the VIBE sequence (Fig. 1). The T1-weighted gradient-echo sequence in two-point Dixon technique 22] provides in- and out-phase as well as water- and fat-saturated images. Images were acquired with $\mathrm{TR}=7.5(\mathrm{~ms})$ and multimodal $\mathrm{TE}=2.4 / 4.8$ (ms) and a flip angle of 10. The voxel size was $1.64 \times 1.64 \times 4.0 \mathrm{~mm}$, and 64 slices were acquired in $19 \mathrm{~s}$. The image resolution used is common for diagnosis in clinical routine although it is relatively coarse. It was used in SHIP in order to avoid long image acquisition times. The Dixon technique ensures that the four different datasets are sufficiently well registrated for the subsequent methods that are used in our liver volumetry framework.

\section{Description of the Method}

\subsection{Denoising of MR Datasets}

Edge-preserving anisotropic diffusion [23] is used to homogenize the MR intensities inside the different tissue types and to preserve edge magnitudes that are important for distinguishing liver tissue from adjacent tissues. We applied this denoising method over 50 iterations by using time steps of 0.0625 .

\subsection{Training Phase}

A training phase is mandatory for supervised probability map generation techniques. Thus, for training purposes, binary masks of 20 livers from different subjects were segmented manually and saved. Manual segmentation was performed by medical experts. The binary masks served to obtain prior knowledge of MR signal intensity distributions and can be used for later probablity map generation. Since many subjects in SHIP have fatty livers, we determined MR signal intensities separately for fatty liver and nonfatty livers. As biopsy results 


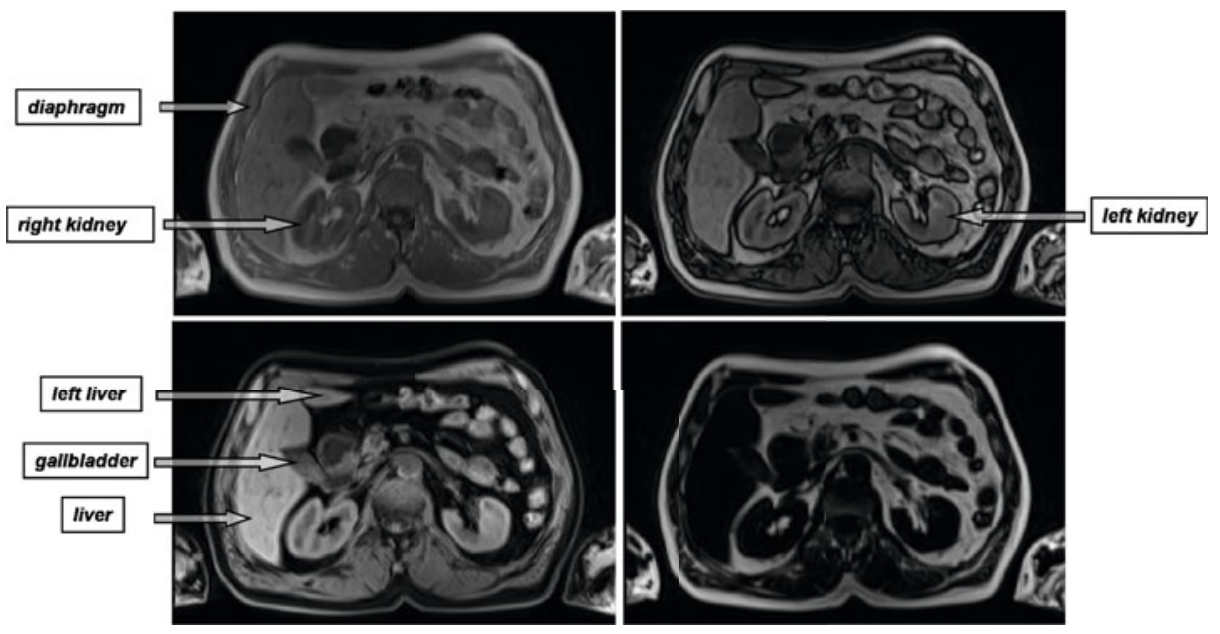

Fig. 1. Original MR images taken from a transverse slice of the VIBE sequence. Inphase weighting (upper left), opposed-phase weighting (upper right), fat-saturated weighting (lower left), water-saturated weighting (lower right). Frequently mentioned organs and tissue types in this work are labeled on the images for better orientation.

are available for some SHIP subjects, we selected MR datasets of 10 subjects with nonfatty livers ( $<5 \%$ fat) and 10 subjects with fatty livers $(25 \%-64 \%$ fat) for this purpose. For our supervised recognition technique, we aim to enhance the transition region between liver and kidney and therefore we have to assign three tissue types (liver, kidney, and background). Hence, binary masks of the 20 selected subjects' right kidneys were segmented manually by medical experts and saved separately. Finally, an overlap region, which is contained in all 20 binary masks of the segmented livers, was determined. We call this region the "reliable common liver" ( $\mathrm{rcl}$ ) region because it is most likely contained in all liver regions of the SHIP subjects.

\subsection{Probability Map Generation}

MR signal intensity distributions for liver and surrounding tissues are estimated from the training data. Since intensity distributions in the MR images vary for fatty and nonfatty livers, two distributions in the rcl region are estimated. Likelihoods for the two tissue types can be determined with the MR intensity distributions of the two types of trained binary masks. Thus, for every tissue type, we can calculate their mean $\left(\mu_{f}, \mu_{n f}\right)$ and covariance matrices $\left(\Sigma_{f}, \Sigma_{n f}\right)$ and estimate their multivariate normal distributions $\left(P_{f}, P_{n f}\right)$. We extract all $N$ test subjects' MR signal intensity samples $\left(V=\left\{\vec{X}_{i} \| \vec{X}_{i} \in \operatorname{rcl}, i=1 . . N\right\}\right)$ inside the rcl region and assign the liver tissue type according to the maximum likelihood classification by using the calculated log-likelihoods: 


$$
\max \left\{\sum_{i=1}^{N} \ln P_{n f}\left(\overrightarrow{X_{i}} \| \mu_{n f}, \Sigma_{n f}\right), \ln P_{f}\left(\overrightarrow{X_{i}} \| \mu_{f}, \Sigma_{f}\right)\right\}
$$

For generating probability maps we apply the work of Gloger et.al. 24] and use linear discriminant reduction techniques based upon the work of [25] and extended by [26] for multiple distribution classes. By using the binary masks produced during the training phase, MR distributions for the two liver tissue types, the kidney, and the background can easily be estimated. Every sample vector of the collected MR distributions consists of four elements representing the four different MR weightings. We distinguish two types of probability map generation: the two-class case (liver, background) and the three-class case (liver, kidney, background). For each case, we perform linear discriminant reduction (LDA) 25] and determine the axis that serves as optimal projection axis for the data to reduce class overlaps after projection. LDA reduces the dimensionality of the samples from $n=4$ to $n=1$ in a linear manner by preserving as much discriminant information between the given class distributions as possible. By projecting the sample vectors of every class onto the projection axis we obtain one-dimensional probability distributions for every class. The distributions are generated using a kernel density estimator on the histogram. We used a value of $\sigma=2.0$ as standard deviation in the gaussian kernel. Additionally, we incorporate the information of the trained positions in the given binary masks of the three classes as independent probabilities into the likelihoods. In the case of three classes this results in:

$$
\begin{gathered}
P(L \mid \vec{V})=\frac{P(\vec{V} \mid L) P_{x y z}(L)}{P(\vec{V} \mid L) P_{x y z}(L)+P(\vec{V} \mid K) P_{x y z}(K)+P(\vec{V} \mid B) P_{x y z}(B)} \\
P_{x y z}(L)=P_{x y z}(L \mid x, y, z)=P(L \mid x) P(L \mid y) P(L \mid z)
\end{gathered}
$$

for the liver class. Here $V=V(x, y, z)$ represents a voxel with its MR dataset coordinate and $L, K, B$ represent the 3 classes of liver, kidney, and background, respectively. Liver pobability map generation for the two-class case is defined straight-forward in the same manner as (2) and (3), treating kidney samples and kidney locations as part of the background class information. In this approach we do not differentiate between the class occurrences and set the a priori probabilities of every class to 1 . For every test subject we calculate the liver probability maps for the three-class case and the two-class case, depending on which liver tissue type is present. The segmentation is performed in the liver probability map of the two-class case, incorporating liver probability maps of the three-class case for refinement purposes (Fig. 2).

\subsection{D Level Set Segmentation}

Instead of using original MR datasets, we perform level set segmentation on the calculated probability maps. We use the liver probability map of the twoclass case calculated as described in the previous section. In the liver probability 

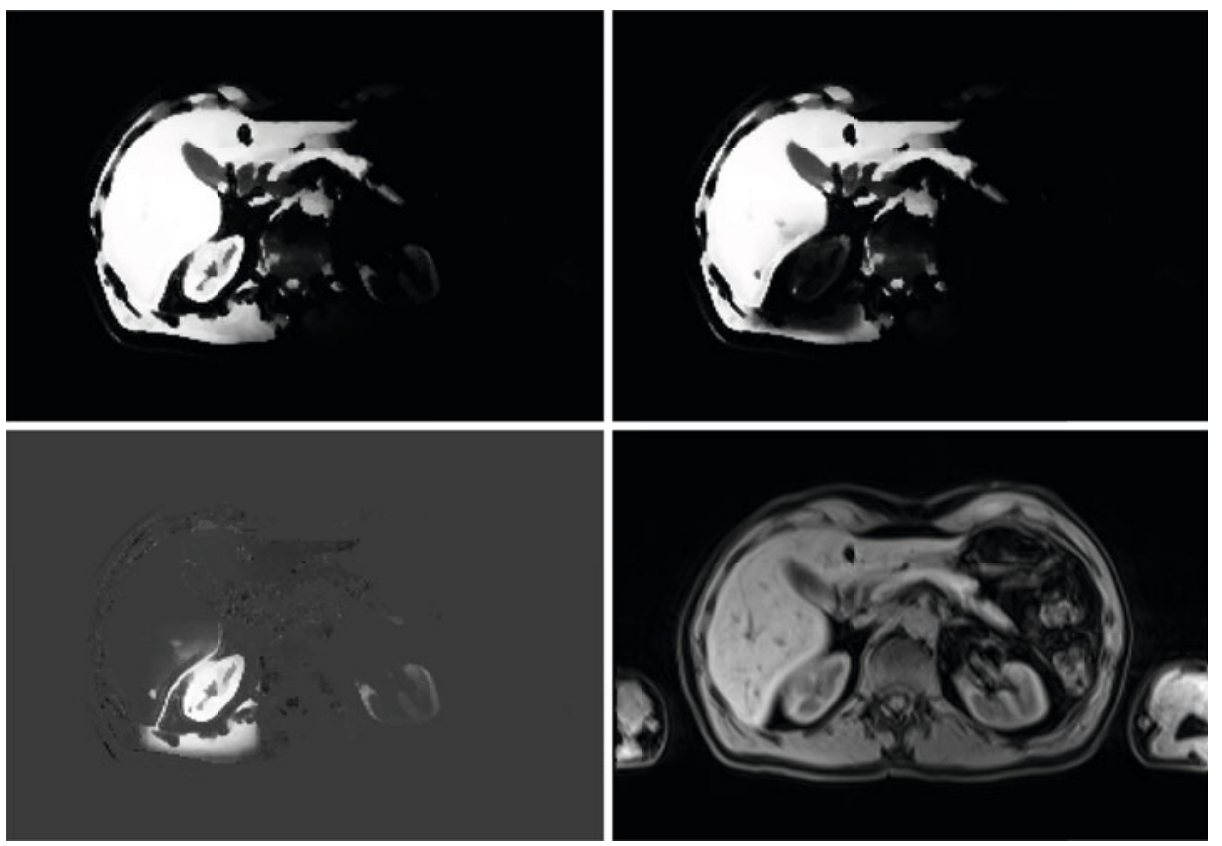

Fig. 2. Two-class liver probability map (upper left) and three-class liver probability map with kidney significantly suppressed (upper right). Difference image enhances transition region between liver and kidney (lower left). Original fat-saturated image (lower right).

map, the most probable liver tissue is enhanced based on liver- specific MR signal intensity distributions and MR dataset locations. A further advantage is that we can use the maximum value of the probability map as the starting point for $3 \mathrm{D}$ level set segmentation. During the test phase of our method, we observed that the maximum value is always located inside the liver. As possible MR artifacts or liver disease may show different probability values, we use the Geodesic Active Contour approach of Caselles et.al. [21]. Consequently, we use the flexibility of the level sets to perform topological changes for our segmentation purpose. A drawback of the level set method is its time-consuming propagation process. Hence, we perform the segmentation in two steps: a fast and coarse segmentation step on lower resolved probability maps and a fine segmentation step in the probablity maps with original resolution. Fine segmentation is initiated with the coarse segmentation result that is scaled up to the original matrix resolution. Level set propagation is steered by the following partial diffential equation:

$$
\frac{\partial \phi}{\partial t}=g(p m)(a+b \kappa)|\nabla \phi|+\nabla g(p m) \nabla \phi
$$

Here, $\phi$ represents the signed distance function depending on the segmenting surface, $\kappa$ is the mean curvature, $p m=\operatorname{pm}(x, y, z)$ represents the probability map values at the MR dataset positions, and $a, b$ are weighting parameters for 
propagation speed and level set mean curvature weighting. As stopping function we chose:

$$
g(p m)=\frac{1}{1+|\nabla p m|^{2}}
$$

The stopping effect of $g$ is influenced by the strength of the probability map gradients. To accelerate level set propagation we suppress the influence of lower probability map gradients. Instead of using a threshold to remove low gradients we use a sigmoid function to transform the gradient values to the relevant range of gradients to ensure faster propagation. We focus on a particular set of gradient values and progressively attenuate values outside that range:

$$
S(|\nabla p m|)=(\max (|\nabla p m|)-\min (|\nabla p m|)) \frac{1}{1+\exp \left(-\frac{|\nabla p m|-\beta}{\alpha}\right)}+\min (|\nabla p m|)
$$

For the sigmoid transformation we apply the following values for the parameters: $\alpha=10$ and $\beta=\frac{\max (|\nabla p m|)}{3}$.

\subsection{Distance Transform Based Segmentation Refinement}

Due to adjacent tissue with similar appearance overspills can occur. Some of those overspills could be avoided by penalizing the curvature term during level set propagation. However, it was crucial to find a representative weighting parameter for penalizing the curvature term for all the variously shaped livers having different curvature properties. In case of strong curvature penalizations, elongated and tapered liver parts are excluded during segmentation. Interior liver parts having low probability values (i.e. vasular structures or the hepatic portal vein) have to be overcome by topological changes. This is not possible, if high curvatures are necessary to overcome those inner structures. Since, higher penalization of the curvature term produces undersegmentations we used curvature weightings, which in fact can produce overspills but includes all liver parts.

Then we applied a new method to remove those overspills, which are recognized and removed in a postprocessing step where we assume that regions erroneously connected to the liver are touching the liver only in a small region. By eroding the liver boundary such regions should be found since erosion should split the segment at those sites. Hence we erode the liver segment, remove erroneously included segment parts and use the remaining, eroded part to predict the correct liver boundary at sites of overspill. We use the distance transform that has been generated by the level set segmentation to guide this erosion process. During level set propagation we have assigned positive distance values inside the liver and negative ones outside the liver. Hence, the positive part represents a distance transform of the segmentation result (SR) and we determine an initial 3D label (SR label) constructed by all positive distance values. Now we iteratively subtract a distance value (we chose $1 \mathrm{~mm}$ ) from the signed distance map, which leads to a subsequent inwards shrinkage of the zero level set. If the segment is split in a shrinkage step, then we continue with the segment that contains the 


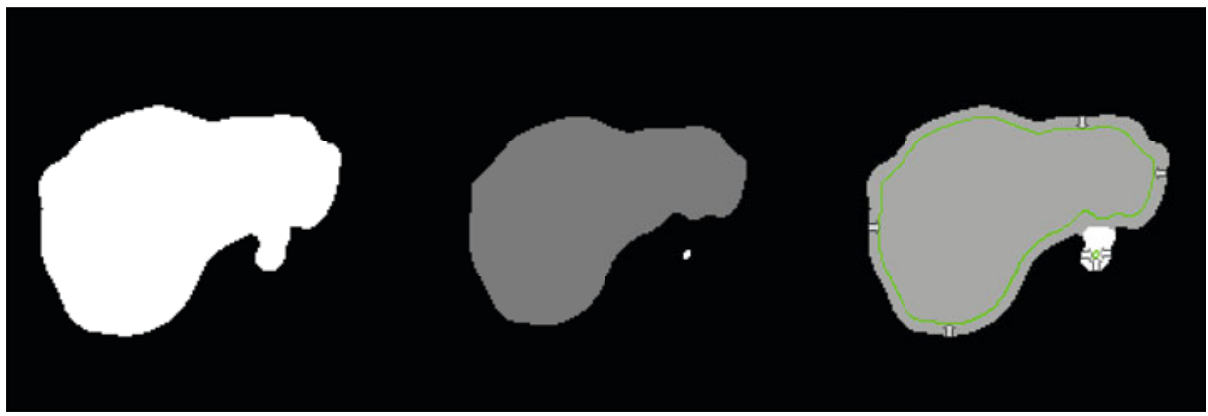

Fig. 3. Slice of 3D label resulting from level set segmentation (left). Same slice following label separation as a result of the shrinkage method (middle). Voxels contained in level set segmentation result and not contained in one of the separated $3 \mathrm{D}$ labels are assigned to the 3D labels according to their minimal euclidean distance. The borders of the shrinked 3D labels (middle) are depicted in green with arrows indicating the shrinkage direction (right).

starting point of the level set propagation. Voxels removed by the actual shrinkage step get segment labels assigned according to their euclidean distance to the nearest segment. We construct a new signed distance map depending on the liver-representing label surface. Thus, we remove all labels which we regard as overspills (Fig. 30).

Given the high liver shape variability and the fact that livers consist of left and right liver parts we aim to avoid label separations inside the liver. Livers can show concave surface regions, which can lead to separations inside the liver when this method is used. We found that most overspills are detected in the first shrinkage steps; hence, we use an upper shrinkage frontier $(20 \mathrm{~mm})$ to avoid removal of liver parts. However, parts of the kidney region may not be removable by this technique since the kidney has similar tissue properties, and there is a higher degree of surface contact between liver and kidney. Overspill into kidney regions can be removed if we perform shrinkage beyond $20 \mathrm{~mm}$ but only at sites where label separations occur inside the transition region between liver and kidney. This transition region can be determined by subtracting the liver probability map of the two-class case from the liver probability map of the three-class case (Fig. 2, lower left). We threshold this difference dataset by choosing the half of the probability map maximum $(0.5$ in case of probability values of $[0 ; 1])$.

\section{Results}

We tested our method on $40 \mathrm{MR}$ datasets from different subjects, which are not contained in the training set. 20 Twenty of the MR datasets contained livers with normal tissue and $20 \mathrm{MR}$ datasets showed livers with fatty tissue. Result quality was different depending on whether fatty livers or nonfatty livers were 
Table 1. Mean (Mean) and standard deviations (Std) of segmentation quality measures for evaluating liver segmentation with the new method proposed here. Segmentation quality differs for nonfatty livers (left) versus fatty livers (right).

\begin{tabular}{l|cccc|cccc} 
& \multicolumn{4}{|c|}{ Nonfatty livers } & \multicolumn{4}{c}{ Fatty livers } \\
& VE & OE & DICE & TPF & VE & OE & DICE & TPF \\
\hline Mean & 0.047 & 0.129 & 0.944 & 0.881 & 0.120 & 0.206 & 0.892 & 0.940 \\
Std & 0.031 & 0.028 & 0.020 & 0.052 & 0.078 & 0.082 & 0.057 & 0.017
\end{tabular}

segmented. Hence, we evaluate and present the results for fatty and nonfatty livers separately (Tab. 1).

We used three segmentation quality measures for result evaluation. We calculate the volume error (with $V_{T}, V_{S}$ representing the training and segmented volume respectively)

$$
V E=\frac{V_{T}-V_{S}}{V_{T}}
$$

and overlap error according to:

$$
O E=\frac{N\left(\left|M_{T}-M_{S}\right|\right)}{N\left(M_{T}\right)}
$$

with $M_{T}, M_{S}$ representing the binary masks of trained and segmented dataset respectively. $N()$ represents the number of voxels. Furthermore, we calculated the true positive fraction according to:

$$
T P F=\frac{N\left(M_{T} \cap M_{S}\right)}{N\left(M_{T}\right)}
$$

Finally, we determined the DICE coefficient, which has been established as a reliable measure for segmentation quality in large-scale studies [27]:

$$
D I C E=\frac{2 N\left(M_{T} \cap M_{S}\right)}{N\left(M_{T}\right)+N\left(M_{S}\right)}
$$

Our method performs better when used to segment nonfatty livers. All quality measures are superior for nonfatty livers compared with fatty livers. A comparison with existing approaches to liver segmentation in $\mathrm{CT}$ and MR datasets is difficult due to inconsistently used quality measures. Unfortunately, reliable data on the quality of MR segmentation is scarce. Although comparing quality measures between different imaging modalilities is precarious, we compare our results with the few results published on CT datasets. In [5 the authors report overlap errors of 12.2 , which is nearly the same as our results for nonfatty livers. The authors in 28] achieved a mean value of 0.91 for the Dice coefficient, which is worse than our results for nonfatty livers. The only reliable information about quality measures for MR liver segmentation is given in [18: our results for the 
TPF measures are with a mean value of 0.94 nearly as good as their TPF results of 0.95. However, our method is fully automatic and takes different tissue types into account, which is not possible by applying the method described by Chen et al. Result analysis with visualization techniques in our volumetry framework shows that probability maps of nonfatty livers are less susceptible to overspill. Furthermore, overspills from probability maps of nonfatty livers are easier to remove. With the upper border for the maximum shrinkage distance we used during the distance-based refinement phase, some overspills are not removed from fatty liver probability maps.

\section{Discussion}

We developed a fully automatic 3D volumetry framework, which uses and extends an existing edge-based level set approach to segment livers in MR datasets. Previously, we tested also a well-known region-based level set approach [29] delivering worse results than our extended edge-based level set approach. A two-step level set segmentation is performed in liver probability maps produced by combining methods of linear discriminant analysis with a Bayesian formulation. We filter relevant liver edges from liver probability maps by using sigmoid functions which generates adapted stopping functions to improve and accelerate level set propagations. Prior knowledge about liver-specific MR intensity distributions is collected during a primary training phase, which is used for probability map generation. Thus, no user-interaction is required. Our method differentiates between fatty liver tissue and non-fatty liver tissue and generates tissue-specific probability maps automatically. Consequently, our method performs liver sgementation for both tissue types, which is very helpful for applications in epidemiological studies.

We tested our method for 40 probands of an epidemiological study and achieved good results. Comparisons show that our results for non-fatty livers are in the same quality range as results from existing approaches for liver segmentation of CT and MR datasets. Our method outperforms several approaches for CT datasets. A volume error of less than $5 \%$ for non-fatty livers is already appropriate for fully automatic non-fatty liver volumetry in large-scale studies.

The segmentation results for fatty livers should be improved to be used for epidemiological studies. Fatty liver tissue shows more similarity to adjacent tissue types, which reduces the probability map quality for the application of edgebased level set segmentation techniques. Due to higher tissue similarity, overspill into adjacent tissue is more probable on fatty liver probability maps. In future work we aim to improve the segmentation quality especially for fatty livers by using more relevant liver features in order to reduce the volume error below $10 \%$. Although livers show high shape variabilities, we will incorporate prior shape information to improve the results for fatty livers. 


\section{References}

[1] Gao, L., Heath, D., Kuszyk, B., Fishman, E.: Automatic liver segmentation technique for three-dimensional visualization of CT data. Radiology 201(2), 359-364 (1996)

[2] Seo, K.S.: Improved Fully Automatic Liver Segmentation Using Histogram Tail Threshold Algorithms. In: International Conference on Computational Science, pp. 822-825 (2005)

[3] Evans, A., Lambrou, T., Linnery, A., Todd-Pokroped, A.: Automatic Segmentation of Liver Using a Toplogy Adaptive Snake. In: International Conference on Biomedical Engineering, pp. 205-208 (2004)

[4] Schenk, A., Prause, G.P.M., Peitgen, H.O.: Efficient semiautomatic segmentation of $3 \mathrm{~d}$ objects in medical images. In: Medical Image Computing and ComputerAssisted Intervention, pp. 186-195 (2001)

[5] Daisuke, F., Akinobu, S., Hidefumi, K.: Automatic Liver Segmentation Method based on Maximum A Posterior Probability Estimation and LevelSet Method. In: Medical Image Computing and Computer Assisted Intervention, pp. 117-124 (2007)

[6] Lamecker, H., Lange, T., Seebass, M.: A Statistical Shape Model for the Liver. In: Dohi, T., Kikinis, R. (eds.) MICCAI 2002. LNCS, vol. 2489, pp. 421-427. Springer, Heidelberg (2002)

[7] Ling, H., Zhou, S.K., Zheng, Y., Georgescu, B., Suehling, M., Comaniciu, D.: Hierarchical, learning-based automatic liver segmentation. In: IEEE Conf. on Computer Vision and Pattern Recognition (CVPR 2008). IEEE Computer Society, Anchorage (2008)

[8] Soler, L., Delingette, H., Malandain, G., Montagnat, J., Ayache, N., Koehle, C., Dourthe, O., Malassagne, B., Smith, M., Mutter, D., et al.: Fully Automatic Anatomical, Pathological, and Functional Segmentation from CT Scans for Hepatic Surgery. In: Hanson, K.M. (ed.), February 14, pp. 246-255. SPIE, San Diego (2000)

[9] Heimann, T., Wolf, I., Meinzer, H.-P.: Active shape models for a fully automated 3D segmentation of the liver - an evaluation on clinical data. In: Larsen, R., Nielsen, M., Sporring, J. (eds.) MICCAI 2006. LNCS, vol. 4191, pp. 41-48. Springer, Heidelberg (2006)

[10] Li, M., Yang, L.: Liver Segmentation Based on Expectation Maximization and Morphological Filters in CT Images. In: Bioinformatics and Biomedical Engineering (ICBBE 2007). IEEE, Wuhan (2007)

[11] Freiman, M., Eliassaf, O., Taieb, Y., Joskowicz, L., Azraq, Y., Sosna, J.: An iterative Bayesian approach for nearly automatic liver segmentation: algorithm and validation. International Journal of Computer Assisted Radiology and Surgery 3(5), 439-446 (2008)

[12] Sosna, J., Berman, P., Azraq, Y., Libson, E.: Liver segmentation and volume calculation from MDCT using Bayesian likelihood maximization technique: comparison with manual tracing technique. RSNA, Chicago (2006)

[13] Massoptier, L., Casciaro, S.: Fully automatic liver segmentation through graphcut technique. In: Rousseau, J., Delhomme, G., Akay, M. (eds.), Lyon, France, August 22-26, pp. 5243-5246. IEEE, Los Alamitos (2007)

[14] Beichel, R., Bauer, C., Bornik, A., Sorantin, E., Bischof, H.: Liver Segmentation in CT Data: A Segmentation Refinement Approach. In: Ayache, N., Ourselin, S., Maeder, A. (eds.), Brisbane, Australia, pp. 235-245. Springer, Heidelberg (October 29, 2007) 
[15] Rusko, L., Bekes, G.: Liver segmentation for contrast-enhanced MR images using partitioned probabilistic model. International Journal of Computer Assisted Radiology and Surgery 6(1), 13-20 (2010)

[16] Platero, C., Gonzalez, M., Tobar, M.C., Poncela, J.M., Sanguino, J., Asensio, G., Santos, E.: Automatic method to segment the liver on multi-phase MRI. In: Jover, J.H. (ed.), Barcelona, Spain, June 25-28 (2008)

[17] Cheng, K., Gu, L., Xu, J.: A novel shape prior based level set method for liver segmentation from MR Images, Shenzhen, China, May 30-31, pp. 144-147 (2008)

[18] Chen, G., Gu, L., Qian, L., Xu, J.: An Improved Level Set for Liver Segmentation and Perfusion Analysis in MRIs. IEEE Transactions on Information Technology in Biomedicine 3(1), 94-103 (2009)

[19] Yuan, Z., Wang, Y., Yang, J., Liu, Y.: A novel automatic liver segmentation technique for MR images. In: Image and Signal Processing (CISP), Yantai, pp. 1282-1286 (2010)

[20] Baumeister, S.E., Voelzke, H., Marschall, P., John, U., Schmidt, C.O., Flessa, S., Alte, D.: Impact of Fatty Liver Disease on Health Care Utilization and Costs in a General Population: A 5-Year Observation. Gastroenterology 134(1), 85-94 (2008)

[21] Caselles, V., Kimmel, R., Sapiro, G.: Geodesic active contours. In: International Conference on Computer Vision (ICCV 1995). IEEE Computer Society, Massachusetts (1995)

[22] Hussain, H.K., Chenevert, T.L., Londy, F.J., Gulani, V., Swanson, S.D., McKenna, B.J., Appelman, H.D., Adusumilli, S., Greenson, J.K., Conjeevaram, H.: Hepatic fat fraction: MR imaging for quantitative measurement and display early experience. Radiology 237, 1048-1055 (2005)

[23] Whitaker, R.T., Xue, X.: Variable-conductance, Level-Set Curvature for Image Denoising, Thessaloniki, Greece, pp. 142-145 (2001)

[24] Gloger, O., Kuehn, J., Stanski, A., Voelzke, H., Puls, R.: A fully automatic threestep liver segmentation method on LDA-based probability maps for multiple contrast MR images. Magnetic Resonance Imaging 28(6), 882-897 (2010)

[25] Fisher, R.: The statistical utilization of multiple measurements. Ann. Eugenics 8, 376-386 (1938)

[26] Rao, C.: The utilization of multiple measurements in problems of biological classification. Journal of the Royal Statistical Society 10, 159-203 (1948)

[27] Crum, W.R., Camara, O., Hill, D.L.G.: Generalized Overlap Measures for Evalution and Validation in Medical Image Analysis. IEEE Transactions on Medical Imaging 25(11), 1451-1458 (2006)

[28] Maier, F., Wimmer, A., Soza, G., Kaftan, J.N., Fritz, D., Dillmann, R.: Automatic Liver Segmentation Using the Random Walker Algorithm. Bildverarbeitung fuer die Medizin, pp. 65-61 (2008)

[29] Chan, T.F., Vese, L.A.: Active contours without edges. IEEE Transactions on Image Processing 10(2), 266-277 (2001) 